ORIGINAL ARTICLE

\section{A 9-Valent HPV Vaccine against Infection and Intraepithelial Neoplasia in Women}

\author{
E.A. Joura, A.R. Giuliano, O.-E. Iversen, C. Bouchard, C. Mao, J. Mehlsen, \\ E.D. Moreira, Jr., Y. Ngan, L.K. Petersen, E. Lazcano-Ponce, P. Pitisuttithum, \\ J.A. Restrepo, G. Stuart, L. Woelber, Y.C. Yang, J. Cuzick, S.M. Garland, W. Huh, \\ S.K. Kjaer, O.M. Bautista, I.S.F. Chan, J. Chen, R. Gesser, E. Moeller, M. Ritter, \\ S. Vuocolo, and A. Luxembourg, for the Broad Spectrum HPV Vaccine Study*
}

A BSTRACT

BACKGROUND

The investigational 9-valent viruslike particle vaccine against human papillomavirus (HPV) includes the HPV types in the quadrivalent HPV (qHPV) vaccine $(6,11,16$, and 18) and five additional oncogenic types (31, 33, 45, 52, and 58). Here we present the results of a study of the efficacy and immunogenicity of the 9vHPV vaccine in women 16 to 26 years of age.

\section{METHODS}

We performed a randomized, international, double-blind, phase $2 \mathrm{~b}-3$ study of the $9 \mathrm{vHPV}$ vaccine in 14,215 women. Participants received the 9vHPV vaccine or the qHPV vaccine in a series of three intramuscular injections on day 1 and at months 2 and 6. Serum was collected for analysis of antibody responses. Swabs of labial, vulvar, perineal, perianal, endocervical, and ectocervical tissue were obtained and used for HPV DNA testing, and liquid-based cytologic testing (Papanicolaou testing) was performed regularly. Tissue obtained by means of biopsy or as part of definitive therapy (including a loop electrosurgical excision procedure and conization) was tested for HPV.

\section{RESULTS}

The rate of high-grade cervical, vulvar, or vaginal disease irrespective of HPV type (i.e., disease caused by HPV types included in the 9vHPV vaccine and those not included) in the modified intention-to-treat population (which included participants with and those without prevalent infection or disease) was 14.0 per 1000 person-years in both vaccine groups. The rate of high-grade cervical, vulvar, or vaginal disease related to HPV-31, 33, 45, 52, and 58 in a prespecified per-protocol efficacy population (susceptible population) was 0.1 per 1000 person-years in the 9vHPV group and 1.6 per 1000 person-years in the qHPV group (efficacy of the 9vHPV vaccine, $96.7 \%$; $95 \%$ confidence interval, 80.9 to 99.8 ). Antibody responses to HPV-6, 11, 16, and 18 were noninferior to those generated by the qHPV vaccine. Adverse events related to injection site were more common in the 9vHPV group than in the qHPV group.

CONCLUSIONS

The 9vHPV vaccine prevented infection and disease related to HPV-31, 33, 45, 52, and 58 in a susceptible population and generated an antibody response to HPV-6, 11,16 , and 18 that was noninferior to that generated by the qHPV vaccine. The $9 \mathrm{vHPV}$ vaccine did not prevent infection and disease related to HPV types beyond the nine types covered by the vaccine. (Funded by Merck; ClinicalTrials.gov number, NCT00543543).
The authors' full names, academic degrees, and affiliations are listed in the Appendix. Address reprint requests to Dr. Joura at the Department of Gynecology and Obstetrics, Medical University of Vienna, Währinger Gürtel 18-20, Vienna, Austria, or at elmar.joura@meduniwien .ac.at.

*A complete list of the investigators for the Broad Spectrum HPV Vaccine Study is provided in the Supplementary Appendix, available at NEJM.org.

N EngIJ Med 2015;372:711-23. DOI: 10.1056/NEJMoal405044 Copyright (C) 2015 Massachusetts Medical Society. 
$\mathrm{T}$ HE HUMAN PAPILLOMAVIRUS (HPV) causes premalignant and malignant lesions of the cervix, ${ }^{1,2}$ vagina, ${ }^{3,4}$ vulva, ${ }^{4,5}$ anus, ${ }^{4,6}$ penis, ${ }^{7}$ and oropharynx, ${ }^{8}$ as well as genital warts. ${ }^{9,10}$ The recent development of prophylactic vaccines directed against the most relevant disease-causing HPV types has helped to prevent diseases related to HPV. ${ }^{11}$

In clinical trials, the bivalent HPV viruslike particle vaccine against HPV types 16 and 18 was efficacious against related infection with these types and against cervical dysplasia, ${ }^{12}$ and the quadrivalent $\mathrm{HPV}$ viruslike particle vaccine against types $6,11,16$, and 18 was efficacious against related infection and against cervical, vaginal, vulvar, and anal dysplasia and against condyloma related to HPV-6 and $11 .{ }^{13-17}$ Post-licensure reports from countries with established HPV vaccination programs indicate that HPV vaccination has a beneficial effect at the population level as early as 3 years after the introduction of an HPV vaccination program, including decreases in the incidence of high-grade cervical abnormalities, ${ }^{18-21}$ the prevalence of vaccine HPV types, ${ }^{22-24}$ and the incidence of genital warts. ${ }^{25-29}$ Analyses of clinical trial and post-licensure safety data have not identified safety concerns associated with HPV vaccination. ${ }^{30-37}$

Current HPV vaccines address approximately $70 \%$ of cervical cancers through protection from HPV-16 and HPV-18. Partial cross-protection against nonvaccine HPV types has been reported for both licensed vaccines, although the clinical significance of the partial cross-protection remains uncertain. ${ }^{38}$ The investigational 9-valent HPV viruslike particle (9vHPV) vaccine addresses the four HPV types $(6,11,16,18)$ that are in the quadrivalent $\mathrm{HPV}$ vaccine plus five additional oncogenic types (31, 33, 45, 52, and 58). The 9vHPV vaccine offers the potential to increase overall prevention of cervical cancer from approximately $70 \%$ to approximately $90 \% .{ }^{2,39,40}$ Here we report the findings of an efficacy and immunogenicity study of the $9 \mathrm{vHPV}$ vaccine.

\section{METHODS}

STUDY DESIGN

We conducted a randomized, international, multicenter, double-blind study of the immunogenicity, efficacy, and side-effect profile of the 9vHPV vaccine in women 16 to 26 years of age. The study was based on a phase 2-3 adaptive design (see the Supplementary Appendix, available with the full text of this article at NEJM.org). An initial group of 1242 women were randomly assigned to receive one of three doses of the 9vHPV vaccine or a qHPV vaccine control. A larger group of 13,598 women were then randomly assigned to receive either the 9vHPV vaccine at the dose selected on the basis of results in the initial group or the $\mathrm{qHPV}$ vaccine control. The efficacy study included these 13,598 women together with the 307 women in the initial group assigned to receive the 9vHPV vaccine at the dose selected and the 310 women in the initial group assigned to the $\mathrm{qHPV}$ vaccine, representing a total of 14,215 women (Fig. S1 and S2 in the Supplementary Appendix).

Since HPV vaccination is widely recommended and has been shown to prevent HPV disease related to oncogenic HPV types, the use of a placebo was not considered to be acceptable for ethical reasons. Consequently, the study used the qHPV vaccine as an active comparator. Participants were eligible if they had no history of an abnormal result on a Papanicolaou (Pap) test, no more than four lifetime sexual partners, and no previous abnormal finding on cervical biopsy.

\section{STUDY OVERSIGHT}

The study was conducted in accordance with principles of Good Clinical Practice and was approved by the institutional review board at each participating institution and by regulatory agencies. Written informed consent was provided by all adult participants and by a parent or legal guardian of participants who were minors. A scientific advisory committee comprising both academic and sponsor (Merck) investigators developed the protocol (available at NEJM.org), formulated the statistical analysis plan, analyzed and interpreted the data, and wrote the manuscript. The external data and safety monitoring committee whose members were aware of the group assignments assessed safety findings throughout the study. All the authors vouch for the completeness and accuracy of the data and analyses presented.

\section{VACCINE DOSING}

A $0.5-\mathrm{ml}$ dose of $\mathrm{qHPV}$ vaccine contains $20 \mu \mathrm{g}$ of HPV-6, $40 \mu \mathrm{g}$ of HPV-11, $40 \mu \mathrm{g}$ of HPV-16, and $20 \mu \mathrm{g}$ of $\mathrm{HPV}-18$ viruslike particles, and 


\begin{tabular}{|c|c|c|c|}
\hline Characteristic & $\begin{array}{l}\text { 9vHPV Vaccine } \\
\qquad(\mathrm{N}=7106)\end{array}$ & $\begin{array}{l}\text { qHPV Vaccine } \\
(\mathrm{N}=7109)\end{array}$ & $\begin{array}{c}\text { Total } \\
(\mathrm{N}=14,215)\end{array}$ \\
\hline \multicolumn{4}{|l|}{ Age $-y r$} \\
\hline Mean & $21.9 \pm 2.5$ & $21.8 \pm 2.5$ & $21.9 \pm 2.5$ \\
\hline Median & 22.0 & 22.0 & 22.0 \\
\hline Range & $16-26$ & $16-26$ & $16-26$ \\
\hline Age at first sexual intercourse $-y r$ & $17.4 \pm 2.2$ & $17.4 \pm 2.2$ & $17.4 \pm 2.2$ \\
\hline \multicolumn{4}{|l|}{ Region - no. (\%) } \\
\hline Asia-Pacific & $905(12.7)$ & $909(12.8)$ & $1,814(12.8)$ \\
\hline Europe & $2406(33.9)$ & $2409(33.9)$ & $4,815(33.9)$ \\
\hline Latin America & $2372(33.4)$ & $2372(33.4)$ & $4,744(33.4)$ \\
\hline North America & $1423(20.0)$ & $1419(20.0)$ & $2,842(20.0)$ \\
\hline \multicolumn{4}{|l|}{ Smoking status - no. (\%) } \\
\hline Current smoker & $1071(15.1)$ & $1005(14.1)$ & $2,076(14.6)$ \\
\hline Former smoker & $382(5.4)$ & $358(5.0)$ & $740(5.2)$ \\
\hline Never smoked & $5647(79.5)$ & $5744(80.8)$ & $11,391(80.1)$ \\
\hline Unknown & $6(0.1)$ & $2(0)$ & $8(0.1)$ \\
\hline \multicolumn{4}{|l|}{ Lifetime sexual partners — no. (\%)† } \\
\hline 1 & $2063(29.0)$ & $2023(28.5)$ & $4,086(28.8)$ \\
\hline 2 & $1691(23.8)$ & $1698(23.9)$ & $3,389(23.8)$ \\
\hline 3 & $1648(23.2)$ & $1646(23.2)$ & $3,294(23.2)$ \\
\hline 4 & $1520(21.4)$ & $1527(21.5)$ & $3,047(21.4)$ \\
\hline$>4$ & $11(0.2)$ & $15(0.2)$ & $26(0.2)$ \\
\hline \multicolumn{4}{|c|}{$\begin{array}{c}\text { Non-HPV-related cervicovaginal infections } \\
\text { or sexually transmitted diseases }\end{array}$} \\
\hline Any & $298(4.2)$ & $292(4.1)$ & $590(4.2)$ \\
\hline Chlamydia & $284(4.0)$ & $285(4.0)$ & $569(4.0)$ \\
\hline Gonorrhea & $19(0.3)$ & $11(0.2)$ & $30(0.2)$ \\
\hline \multicolumn{4}{|l|}{ Contraceptive use $\downarrow$} \\
\hline Barrier & $2318(32.6)$ & $2303(32.4)$ & $4,621(32.5)$ \\
\hline Behavior & $1014(14.3)$ & $1035(14.6)$ & $2,049(14.4)$ \\
\hline Hormonal & $4273(60.2)$ & $4292(60.4)$ & $8,565(60.3)$ \\
\hline \multicolumn{4}{|l|}{$\begin{array}{l}\text { Day } 1 \text { composite HPV positivity - no./ } \\
\text { total no. (\%) } \mathbb{}\end{array}$} \\
\hline Serologic test & $2771 / 7082(39.1)$ & $2647 / 7078(37.4)$ & $5418 / 14,160(38.3)$ \\
\hline PCR assay & $1887 / 6919(27.3)$ & $1920 / 6943(27.7)$ & $3807 / 13,862(27.5)$ \\
\hline Serologic test or PCR assay & $3365 / 6970(48.3)$ & $3345 / 6983$ (47.9) & $6710 / 13,953(48.1)$ \\
\hline
\end{tabular}

* Plus-minus values are means \pm SD. The baseline characteristics of the two study groups were similar. The quadrivalent human papillomavirus (HPV) vaccine (qHPV) targets HPV types $6,11,16$, and 18; the 9-valent viruslike particle vaccine (9vHPV) targets the HPV types in the $\mathrm{GHPV}$ vaccine and five additional oncogenic types $(31,33,45,52$, and 58$)$. PCR denotes polymerase chain reaction.

$\dagger$ The percentages for the number of lifetime sexual partners were calculated on the basis of the number of participants for whom there were data on sexual history at enrollment (7102 in the 9vHPV group and 7108 in the qHPV group).

$\lceil$ Participants may have used more than one contraceptive method. A participant is counted once within a category and may be counted in more than one category. The percentages for the numbers of participants who used contraceptives were based on the number for whom this information was available (7102 in the 9vHPV group and 7104 in the qHPV group).

$\int$ Positivity was defined as an anti-HPV titer on immunoassay of at least 30,16, 20,24, 10, 8, 8, 8, and 8 for HPV types 6 , $11,16,18,31,33,45,52$, and 58 , respectively. The numerator in this category represents the number of HPV-positive participants, and the denominator the total number of participants with assay results that could be evaluated. 
Table 2. Effect of 9vHPV Vaccine on the Incidence of Cervical, Vulvar, and Vaginal Disease and of Persistent HPV-Related Infection.*

\begin{tabular}{|c|c|c|c|c|c|}
\hline \multirow[t]{2}{*}{ End Point } & \multicolumn{2}{|c|}{$\begin{array}{l}\text { 9vHPV Vaccine } \\
\quad(\mathrm{N}=7099)\end{array}$} & \multicolumn{2}{|c|}{$\begin{array}{l}\text { qHPV Vaccine } \\
(\mathrm{N}=7105)\end{array}$} & \multirow[t]{2}{*}{$\begin{array}{l}\text { Risk Reduction } \\
\quad(95 \% \mathrm{Cl})\end{array}$} \\
\hline & no./total no. & $\begin{array}{l}\text { cases } / 1000 \\
\text { person- }-\mathrm{r}\end{array}$ & no./total no. & $\begin{array}{l}\text { cases } / 1000 \\
\text { person- } y r\end{array}$ & \\
\hline \multicolumn{6}{|l|}{ Modified intention-to-treat population } \\
\hline \multicolumn{6}{|l|}{ High-grade cervical, vulvar, and vaginal disease $\dagger^{\dagger}$} \\
\hline All participants & $340 / 7027$ & 14.0 & $344 / 7027$ & 14.0 & $0.7(-15.7$ to 14.8$)$ \\
\hline HPV-uninfected on day 1 & $26 / 3032$ & 2.4 & $46 / 3077$ & 4.2 & 42.5 (7.9 to 65.9$)$ \\
\hline Not related to 9 vaccine HPV typest & $26 / 3032$ & 2.4 & $33 / 3077$ & 3.0 & $19.7(-34.5$ to 52.5$)$ \\
\hline Related to 9 vaccine HPV types & $0 / 3032$ & 0.0 & $13 / 3076$ & 1.2 & 100 (70.4 to 100$)$ \\
\hline HPV-infected on day 1 & $314 / 3995$ & 23.1 & $298 / 3950$ & 22.1 & $-4.8(-23.3$ to 10.8$)$ \\
\hline Not related to 9 vaccine HPV typest & $141 / 3995$ & 10.0 & $137 / 3950$ & 9.8 & $-2.0(-30.0$ to 19.9$)$ \\
\hline Related to 9 vaccine HPV types & $173 / 3992$ & 12.4 & $161 / 3946$ & 11.6 & $-6.8(-33.2$ to 14.3$)$ \\
\hline Average risk reduction $\mathbb{\int}$ & - & - & - & - & $19.0(-1.6$ to 35.3$)$ \\
\hline \multicolumn{6}{|c|}{$\begin{array}{l}\text { High-grade cervical epithelial neoplasia, adenocarcino- } \\
\text { ma in situ, and cervical cancer }\end{array}$} \\
\hline All participants & $325 / 6882$ & 14.1 & $326 / 6871$ & 14.1 & $-0.3(-17.3$ to 14.3$)$ \\
\hline HPV-uninfected on day 1 & $26 / 2976$ & 2.5 & $44 / 3009$ & 4.2 & 39.7 (1.8 to 64.3$)$ \\
\hline Not related to 9 vaccine HPV typest & $26 / 2976$ & 2.5 & $31 / 3009$ & 3.0 & $14.3(-49.1$ to 49.1$)$ \\
\hline Related to 9 vaccine HPV types & $0 / 2976$ & 0.0 & $13 / 3009$ & 1.2 & $100(70.3$ to 100$)$ \\
\hline HPV-infected on day 1 & $299 / 3906$ & 23.3 & $282 / 3862$ & 22.2 & $-5.3(-24.1$ to 10.8$)$ \\
\hline Not related to 9 vaccine HPV typest & $131 / 3906$ & 10.1 & $132 / 3862$ & 10.3 & $1.8(-26.0$ to 23.5$)$ \\
\hline Related to 9 vaccine HPV types: & $168 / 3906$ & 13.0 & $150 / 3862$ & 11.7 & $-11.3(-39.6$ to 11.0$)$ \\
\hline Average risk reduction $\mathbb{S}$ & - & - & - & - & $17.1(-4.2$ to 34.0$)$ \\
\hline \multicolumn{6}{|l|}{ Per-protocol efficacy population } \\
\hline \multicolumn{6}{|l|}{ High-grade cervical, vulvar, and vaginal disease $\dagger$} \\
\hline Related to HPV-31, $33,45,52$, or 58 & $1 / 6016$ & 0.1 & $30 / 6,017$ & 1.6 & 96.7 (80.9 to 99.8 ) \\
\hline Related to HPV-6, 11,16 , or 18 & $1 / 5883$ & 0.1 & $3 / 5898$ & 0.2 & $66.6(-203.0$ to 98.7$)$ \\
\hline \multicolumn{6}{|c|}{$\begin{array}{c}\text { High-grade cervical epithelial neoplasia, adenocarcino- } \\
\text { ma in situ, and cervical cancer }\end{array}$} \\
\hline Related to HPV-31, $33,45,52$, or 58 & $1 / 5948$ & 0.1 & $27 / 5943$ & 1.5 & $96.3(79.5$ to 99.8$)$ \\
\hline Related to HPV-6, 11,16 , or 18 & $1 / 5823$ & 0.1 & $1 / 5832$ & 0.1 & $-0.4(\leq-999$ to 97.4$)$ \\
\hline \multicolumn{6}{|l|}{ Persistent infection $\geq 6$ months' duration } \\
\hline Related to HPV-31, $33,45,52$, or 58 & $35 / 5939$ & 2.1 & $810 / 5953$ & 52.4 & 96.0 (94.4 to 97.2$)$ \\
\hline Related to HPV-6, 11,16 , or 18 & $59 / 5812$ & 3.6 & $80 / 5830$ & 5.0 & $26.4(-4.3$ to 47.5$)$ \\
\hline
\end{tabular}

* The total number of participants $(\mathrm{N})$ includes those who received at least one dose of a study vaccine; the no./total no. refers to the number of participants with an end point among the participants who received at least one dose of a study vaccine and had at least one followup visit. The modified intention-to-treat population consisted of participants who received at least one dose of vaccine and for whom there was at least one measurement of efficacy for the end point being analyzed. The per-protocol efficacy population consisted of participants who received all three doses of vaccine within 1 year, were HPV-uninfected (i.e., were seronegative at day $l$ and had negative results on PCR assays for all HPV types tested from day 1 through month 7) to the vaccine HPV type being analyzed, and had no protocol violations. $\mathrm{Cl}$ denotes confidence interval.

$\uparrow$ This category includes high-grade cervical epithelial neoplasia, adenocarcinoma in situ, cervical cancer, high-grade vulvar intraepithelial neoplasia, high-grade vaginal intraepithelial neoplasia, vulvar cancer, and vaginal cancer.

$t$ The nine vaccine HPV types are $6,11,16,18,31,33,45,52$, and 58. Participants with end-point conditions related to the nine vaccine HPV types were those who at any time during the study received a diagnosis of the indicated disease related to any of the nine HPV types included in the vaccine and, in addition, did not receive a diagnosis of the indicated disease at any time during the study that was not related to one of the nine HPV types included in the vaccine. Participants with end-point conditions were counted only once and in only one of these two categories. The sum of case counts in these two categories is equal to the number of end-point cases irrespective of HPV type.

$\int$ The risk reduction shown represents the sample-size-weighted average of the risk reduction in the HPV-uninfected and the HPV-infected subgroups. The HPV-uninfected subgroup consisted of participants who had the following characteristics on day l: a negative finding for squamous intraepithelial lesions, a seronegative finding for the nine vaccine-HPV types, and negative results on PCR assays for all HPV types tested during the study (types $6,11,16,18,31,33,35,39,45,51,52,56,58$, and 59). The HPV-infected subgroup consisted of all participants who were not in the HPV-infected subgroup.

ๆ Persistent infection was defined as detection of the same HPV type in a genital swab or tissue specimen collected on two or more consecutive visits, with an interval of at least 6 months ( \pm 1 month) between the visits. 
$225 \mu \mathrm{g}$ of the adjuvant amorphous aluminum hydroxyphosphate sulfate (AAHS). ${ }^{14}$ A $0.5-\mathrm{ml}$ dose of $9 \mathrm{vHPV}$ vaccine contains $30 \mu \mathrm{g}$ of HPV-6, $40 \mu \mathrm{g}$ of HPV-11, $60 \mu \mathrm{g}$ of HPV-16, $40 \mu \mathrm{g}$ of HPV-18, $20 \mu \mathrm{g}$ of HPV-31, $20 \mu \mathrm{g}$ of HPV-33, 20 $\mu \mathrm{g}$ of HPV-45, $20 \mu \mathrm{g}$ of HPV-52, and $20 \mu \mathrm{g}$ of HPV-58 viruslike particles, and $500 \mu \mathrm{g}$ of AAHS. Vaccines were administered as a $0.5-\mathrm{ml}$ intramuscular injection in three doses, on day 1 and at month 2 and month 6. Information on randomization to a vaccine group is available in the Supplementary Appendix. At study vaccination visits, all participants received a vaccination report card on which they recorded oral temperatures on each of the 5 days after vaccination and adverse events related to the injection site as well as systemic adverse events on each of the 15 days after vaccination.

\section{FOLLOW-UP}

Swabs of labial, vulvar, perineal, perianal, endocervical, and ectocervical tissue and Pap test (ThinPrep; Hologic) samples were collected on day 1 and at months 7, 12, 18, 24, 30, 36, 42, 48, and 54. Swabs were tested for HPV types 6, 11, $16,18,31,33,35,39,45,51,52,56,58$, and 59 by means of a polymerase-chain-reaction (PCR) assay to identify participants who had an active HPV infection at enrollment and to determine end points for HPV infection. Participants with an abnormal result on a Pap test were referred for colposcopy according to a protocol-mandated triage algorithm (Fig. S3 in the Supplementary Appendix). Histologic sections were first reviewed for clinical management by pathologists at a central laboratory (Diagnostic Cytology Laboratories) who were unaware of the patient's treatmentgroup assignment and HPV status and were then reviewed for the determination of end points by an adjudication panel of four pathologists who were unaware of the vaccination assignment.

\section{PRIMARY HYPOTHESES AND END POINTS}

The primary efficacy hypothesis was that, as compared with the qHPV vaccine, the 9vHPV vaccine would reduce the combined incidence of several conditions related to HPV-31, 33, 45, 52, and 58 in women 16 to 26 years of age who were seronegative on day 1 and for whom there were negative results on PCR assays for the relevant HPV type from day 1 through month 7. These conditions (the study end points) were high-grade cervical intraepithelial neoplasia, adenocarcinoma in situ, invasive cervical carcinoma, high-grade vulvar intraepithelial neoplasia, high-grade vaginal intraepithelial neoplasia, vulvar cancer, and vaginal cancer. Determination of the end points was based on a consensus diagnosis, by a panel of at least two pathologists, of high-grade cervical, vulvar, or vaginal disease and the detection of HPV-31, 33, 45, 52, or 58 DNA in an adjacent histologic section of the same biopsy specimen. ${ }^{14}$

It was anticipated that the 9vHPV vaccine and the qHPV vaccine would be similarly efficacious in preventing disease related to HPV-6, 11, 16, and 18. Thus, it was determined that a direct comparison of the two vaccines on the basis of disease end points related to HPV-6, 11, 16, and 18 would be prohibitive in terms of study size. The primary analysis of 9vHPV vaccine efficacy with respect to end points related to HPV-6, 11, 16 , and 18 was designed to determine whether 9vHPV was noninferior to qHPV with respect to immunogenicity. The primary immunogenicity hypothesis was that geometric mean titers for anti-HPV-6, 11, 16, and 18 in the 9vHPV vaccine group would be noninferior to the geometric mean titers in the qHPV vaccine group (as quantified with the use of a competitive Luminex immunoassay ${ }^{41}$.

\section{STATISTICAL ANALYSIS}

The primary efficacy hypothesis was evaluated in the per-protocol efficacy population, which consisted of participants who received all three doses of vaccine within 1 year, did not have the HPV type being analyzed (i.e., were seronegative on day 1 and PCR-negative from day 1 through month 7), and had no protocol violations. Successful demonstration of the primary efficacy hypothesis required the lower boundary of the twosided $95 \%$ confidence interval of vaccine efficacy to be greater than $25 \%$, where vaccine efficacy, or percent risk reduction, was calculated as $100 \times(1$-incidence rate of types in $9 \mathrm{vHPV} \div$ incidence rate of types in $\mathrm{qHPV})$. The $95 \%$ confidence interval of vaccine efficacy was calculated with the use of a binomial, distribution-based exact method. ${ }^{42}$ With further assumptions relating to end-point accrual rate, losses to follow-up, and exclusion rate from the per-protocol efficacy population, we estimated that enrollment of approximately 7000 participants per group would be needed for the phase 3 efficacy study. 


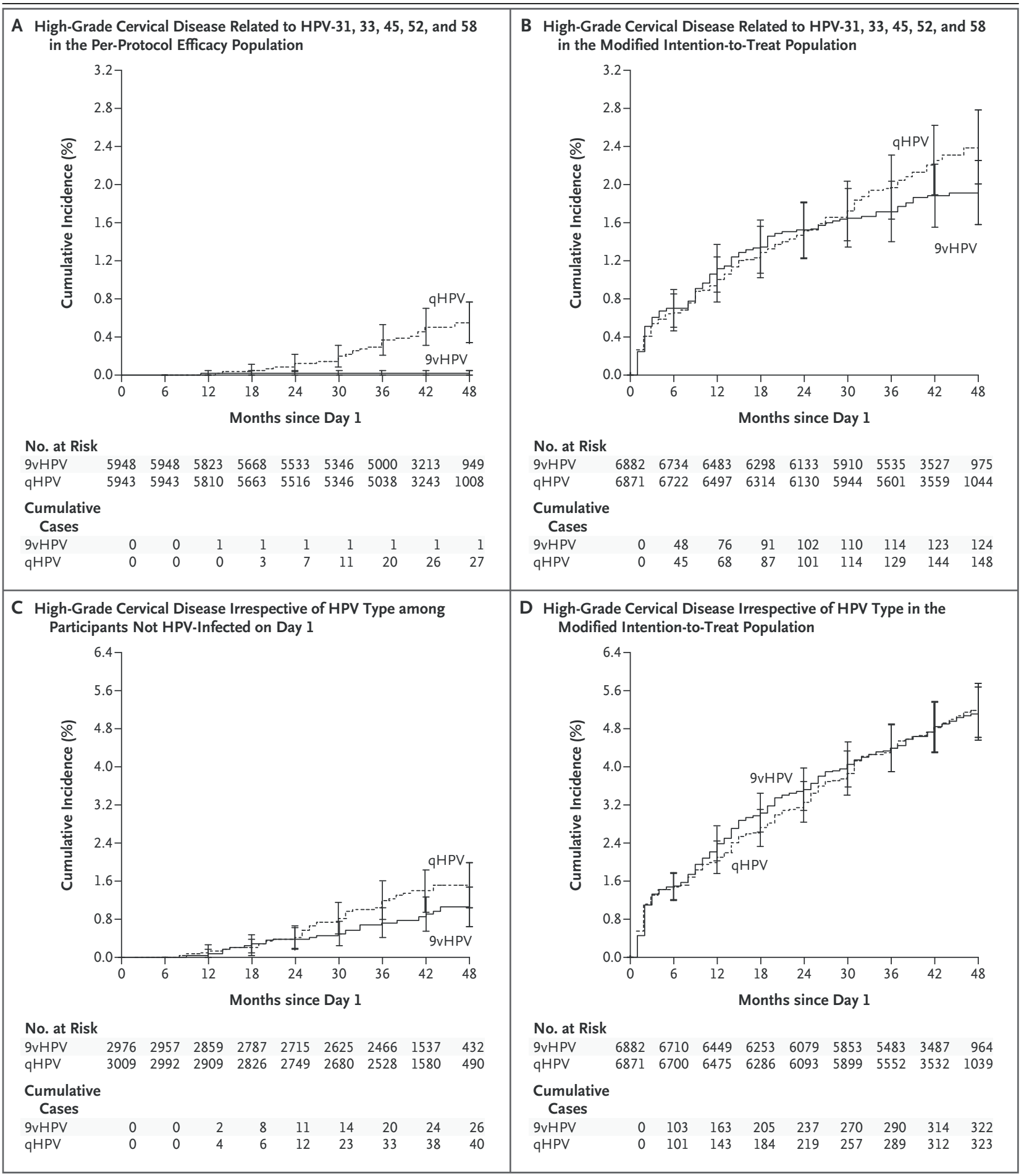

The study used a fixed-event design whereby show a lower boundary of the 95\% confidence the primary efficacy analysis would be performed interval for the estimate of vaccine efficacy of when at least 30 primary efficacy end-point events more than $25 \%$, with a one-sided type I error had been observed. With at least 30 such events, rate of 0.025 , assuming that the true efficacy of the study would have power of $90 \%$ or more to the $9 \mathrm{vHPV}$ vaccine relative to $\mathrm{qHPV}$ vaccine is $83 \%$. 
Figure 1 (facing page). Time to the Development of High-Grade Cervical Disease.

High-grade cervical disease was defined as grade 2 or 3 cervical intraepithelial neoplasia or adenocarcinoma in situ. Data are shown for high-grade cervical disease related to human papillomavirus (HPV) types 31,33 , 45,52 , and 58 in the per-protocol efficacy population (Panel A) and the modified intention-to-treat population (Panel B). Data are also shown for high-grade cervical disease associated with HPV irrespective of the HPV type (i.e., vaccine-type or nonvaccine-type HPV) among participants who were HPV-uninfected on day $l$ (Panel C) and in the modified intention-to-treat population (Panel D). The per-protocol efficacy population (the susceptible population) included participants who received all three doses of vaccine within 1 year, were seronegative on day 1 of the study, and had negative results on polymerase-chain-reaction (PCR) assays of the HPV type being analyzed from day 1 through month 7. The modified intention-to-treat population included all participants who underwent randomization, received at least one dose of vaccine, and had at least one measurement of efficacy; those who had prevalent cervical disease or infection with vaccine-type or nonvaccine-type HPV before study vaccination were not excluded. The HPV-uninfected population included those who received at least one dose of vaccine and who on day 1 had negative results on PCR assays for 14 HPV types, were seronegative for the 9 HPV types in the study vaccine, and had negative results for squamous intraepithelial lesions on Pap tests. The 9-valent HPV (9vHPV) vaccine is a recombinant vaccine against infection with HPV-6, 11 , $16,18,31,33,45,52$, and 58 . The quadrivalent HPV ( $\mathrm{GHPV}$ ) vaccine is a recombinant vaccine against infection with HPV-6, 11, 16, and 18. I bars indicate $95 \%$ confidence intervals.

Case accrual was monitored by a statistician who was independent from the study team and aware of group assignments. No interim efficacy analysis was planned or conducted.

Supportive efficacy analyses were performed in the modified intention-to-treat population, which included participants who received at least one dose of vaccine and for whom there was at least one measurement of efficacy for the corresponding end point. The modified intentionto-treat population included participants who were not HPV-infected at the time of vaccination and participants who were HPV-infected at the time of vaccination. The protocol-specified population of participants who were not HPV-infected included participants who on day 1 had no squamous intraepithelial lesions according to the results of a Pap test, were seronegative for the $9 \mathrm{HPV}$ types in the 9vHPV vaccine, and had negative results on PCR assays for the HPV types tested during the study (HPV-6, 11, 16, 18, 31, $33,35,39,45,51,52,56,58$, and 59). Participants with an HPV-related disease on day 1 were not excluded from the modified intention-totreat population. The estimate of average risk reduction in the modified intention-to-treat population was calculated as the sample-size-weighted average of the percent risk reduction in the subgroup of the participants who were not HPVinfected and in the subgroup of participants who were HPV-infected. The sample-size-weighted average reduction in risk approximates the efficacy expected among participants with characteristics representing the "average" from the two subgroups.

The primary immunogenicity hypothesis was evaluated in the per-protocol immunogenicity population, which consisted of participants eligible for inclusion in the per-protocol efficacy population who received three doses of vaccine during prespecified visit intervals and from whom the 7-month serum sample was obtained within a prespecified interval. Successful demonstration of the primary immunogenicity hypothesis of noninferiority required the lower boundary of the two-sided $95 \%$ confidence interval of the ratio of the geometric mean titer (9vHPV:qHPV) to be greater than 0.67 for each of the anti-HPV types $6,11,16$, and 18 . In separate analyses of each anti-HPV type, the $95 \%$ confidence interval of the ratio for the geometric mean titer was derived from an analysis of variance model with log antiHPV as the response and the vaccination group as the fixed effect. With the planned sample size, this study had a power of more than $99 \%$ to determine a ratio (9vHPV:qHPV) of the true geometric mean titer of at least 0.80 in a test of the primary immunogenicity hypothesis for HPV types $6,11,16$, and 18 .

\section{RESULTS}

\section{STUDY POPULATION}

A total of 14,215 participants underwent randomization for the efficacy portion of this study (Fig. S1 in the Supplementary Appendix). The makeup of the populations for the efficacy and immunogenicity analyses is shown in Table $\mathrm{S} 1$ in the Supplementary Appendix. The baseline characteristics were similar in the two vaccination groups (Table 1). 


\begin{tabular}{|c|c|c|c|c|c|}
\hline \multirow[t]{2}{*}{ Anti-HPV Type } & \multicolumn{2}{|c|}{$\begin{array}{l}\text { 9vHPV Vaccine } \\
\qquad(\mathrm{N}=6792)\end{array}$} & \multicolumn{2}{|c|}{$\begin{array}{c}\text { qHPV Vaccine } \\
(\mathrm{N}=6795)\end{array}$} & \multirow[t]{3}{*}{$\begin{array}{l}\text { GMT Ratio } \\
(95 \% \mathrm{Cl})\end{array}$} \\
\hline & Participants & GMT & Participants & GMT & \\
\hline & no. & $m M U / m l$ & no. & $\mathrm{mMU} / \mathrm{ml}$ & \\
\hline Anti-HPV-6 & 3993 & 893.1 & 3975 & 875.2 & 1.02 (0.99 to 1.06$)$ \\
\hline Anti-HPV-11 & 3995 & 666.3 & 3982 & 830.0 & $0.80(0.77$ to 0.83$)$ \\
\hline Anti-HPV-16 & 4032 & 3131.1 & 4062 & 3156.6 & 0.99 (0.96 to 1.03 ) \\
\hline Anti-HPV-18 & 4539 & 804.6 & 4541 & 678.7 & 1.19 (1.14 to 1.23$)$ \\
\hline \multirow[t]{3}{*}{ Anti-HPV Response } & \multicolumn{2}{|c|}{$\begin{array}{l}\text { 9vHPV Vaccine } \\
\qquad(\mathrm{N}=6792)\end{array}$} & \multicolumn{2}{|c|}{$\begin{array}{l}\text { qHPV Vaccine } \\
\quad(\mathrm{N}=6795)\end{array}$} & $\begin{array}{l}\text { Difference } \\
(95 \% \mathrm{Cl})\end{array}$ \\
\hline & Participants & Seroconversion & Participants & Seroconversion & \\
\hline & no. & no. (\%) & no. & no. (\%) & percentage points \\
\hline HPV-6 cLIA $\geq 30 \mathrm{mMU} / \mathrm{ml}$ & 3993 & $3985(99.8)$ & 3975 & $3969(99.8)$ & $0(-0.3$ to 0.2$)$ \\
\hline HPV-11 cLIA $\geq 16 \mathrm{mMU} / \mathrm{ml}$ & 3995 & $3994(100)$ & 3982 & 3980 (99.9) & $0(-0.1$ to 0.2$)$ \\
\hline HPV- 16 cLIA $\geq 20 \mathrm{mMU} / \mathrm{ml}$ & 4032 & $4031(100)$ & 4062 & $4060(100)$ & $0(-0.1$ to 0.2$)$ \\
\hline HPV-18 cLIA $\geq 24 \mathrm{mMU} / \mathrm{ml}$ & 4539 & $4532(99.8)$ & 4541 & $4528(99.7)$ & $0.1(-0.1$ to 0.4$)$ \\
\hline
\end{tabular}

* The per-protocol immunogenicity population included participants in the per protocol efficacy population who received three doses of vaccine during prespecified visit intervals and from whom the 7-month serum sample was obtained within a prespecified interval. $\mathrm{P}<0.001$ for all comparisons between the 9vHPV vaccine and the $\mathrm{GHPV}$ vaccine. A test of the noninferiority of the 9vHPV vaccine to the $\mathrm{qHPV}$ vaccine with respect to GMT required the demonstration that the GMT with the 9vHPV vaccine was lower than the GMT with the qHPV vaccine by no more than a factor of 1.5 or, equivalently, that the lower boundary of the $95 \%$ confidence interval of the GMT ratio (9vHPV:qHPV) was greater than 0.67. The GMT, GMT ratio, and P values for the between-group differences in GMT were calculated with the use of analysis-ofvariance models. A successful noninferiority test for seroconversion required the demonstration of a statistically significant difference between $9 \mathrm{vHPV}$ and qHPV of no more than 5 percentage points or, equivalently, a lower boundary of the $95 \%$ confidence interval for the difference in percentages that was greater than $-5 \%$. The $95 \%$ confidence interval for the difference and the $\mathrm{P}$ values for seroconversion were calculated with the use of the Miettinen and Nurminen method. The term cLIA denotes competitive Luminex immunoassay, and mMU milli-Merck units (arbitrary units, defined in the Supplementary Appendix).

\section{INCIDENCE OF DISEASE}

Modified intention-to-treat efficacy analyses were performed at baseline on data from participants who were HPV-negative and those who were HPV-positive (according to PCR assays and serologic testing) (Table 2). The incidence of highgrade cervical, vulvar, and vaginal disease among all participants, irrespective of results on HPV testing, was 14.0 per 1000 person-years in both the 9vHPV group and the qHPV group. The rates in the subgroup that was not HPV-infected were 2.4 in the 9vHPV group and 4.2 in the qHPV group (efficacy of the 9vHPV vaccine, 42.5\%; $95 \%$ confidence interval [CI], 7.9 to 65.9$)$. In that subgroup, the efficacy for disease related to the vaccine HPV types was 100\% (95\% CI, 70.4 to 100 ), and the efficacy for disease not related to the vaccine HPV types was $19.7 \%$ (95\% CI, -34.5 to 52.5). The rates among participants who were HPV-infected were similar in the two groups
(23.1 in the 9vHPV vaccine group and 22.1 in the qHPV vaccine group). Further analyses are presented in Table S2 in the Supplementary Appendix.

In the per-protocol efficacy population, the incidence rate of high-grade disease related to HPV-31, 33, 45, 52, and 58 was 0.1 per 1000 person-years in the 9vHPV group and 1.6 per 1000 person-years in the qHPV group (1 case vs. 30 cases; $9 \mathrm{vHPV}$ efficacy, $96.7 \%$; $95 \% \mathrm{CI}, 80.9$ to 99.8) (Table 2). Efficacy analyses according to HPV types are shown in Table S2 in the Supplementary Appendix. The single participant with HPV-58-positive grade 2 cervical epithelial neoplasia in the 9vHPV vaccine group had positive results for HPV-56 at baseline and in all specimens obtained between day 1 and the time of diagnosis, with HPV-58 detected only at the time of diagnosis. In contrast, the incidences of highgrade cervical, vulvar, and vaginal disease in the qHPV group continued to increase over time 


\begin{tabular}{|c|c|c|}
\hline \multirow[t]{2}{*}{ Event } & $\begin{array}{l}\text { 9vHPV Vaccine } \\
\quad(\mathrm{N}=7071)\end{array}$ & $\begin{array}{c}\text { qHPV Vaccine } \\
(\mathrm{N}=7078)\end{array}$ \\
\hline & \multicolumn{2}{|c|}{ no. of participants (\%) } \\
\hline Participants with one or more adverse events $\dagger$ & $6640(93.9)$ & 6419 (90.7) \\
\hline Injection-site event & $6414(90.7)$ & $6012(84.9)$ \\
\hline Pain $\sqrt{ }$ & $6356(89.9)$ & $5910(83.5)$ \\
\hline Mild & $3754(53.1)$ & $4043(57.1)$ \\
\hline Moderate & $2300(32.5)$ & $1682(23.8)$ \\
\hline Severe & $302(4.3)$ & $185(2.6)$ \\
\hline Swelling & $2830(40.0)$ & $2035(28.8)$ \\
\hline Mild: 0 to $\leq 2.5 \mathrm{~cm}$ & $1958(27.7)$ & $1594(22.5)$ \\
\hline Moderate: $>2.5 \mathrm{~cm}$ to $\leq 5.0 \mathrm{~cm}$ & $597(8.4)$ & $332(4.7)$ \\
\hline Severe: $>5.0 \mathrm{~cm}$ & $272(3.8)$ & $109(1.5)$ \\
\hline Unknown & $3(0)$ & $0(0)$ \\
\hline Erythema & $2407(34.0)$ & $1810(25.6)$ \\
\hline Mild: 0 to $\leq 2.5 \mathrm{~cm}$ & $1921(27.2)$ & $1555(22.0)$ \\
\hline Moderate: $>2.5 \mathrm{~cm}$ to $\leq 5.0 \mathrm{~cm}$ & $370(5.2)$ & $197(2.8)$ \\
\hline Severe: $>5 \mathrm{~cm}$ & $114(1.6)$ & $57(0.8)$ \\
\hline Unknown & $2(0)$ & $1(0)$ \\
\hline Pruritus $\rrbracket$ & $388(5.5)$ & $282(4.0)$ \\
\hline Mild & $301(4.3)$ & $223(3.2)$ \\
\hline Moderate & $80(1.1)$ & $56(0.8)$ \\
\hline Severe & $7(0.1)$ & $3(0)$ \\
\hline Systemic event 9 & $3948(55.8)$ & $3883(54.9)$ \\
\hline Any vaccine-related systemic event & $2086(29.5)$ & $1929(27.3)$ \\
\hline Headache & $1031(14.6)$ & $969(13.7)$ \\
\hline Pyrexia & $357(5.0)$ & $301(4.3)$ \\
\hline Nausea & $311(4.4)$ & $261(3.7)$ \\
\hline Dizziness & $211(3.0)$ & $197(2.8)$ \\
\hline Fatigue & $166(2.3)$ & $150(2.1)$ \\
\hline Serious event & $233(3.3)$ & $183(2.6)$ \\
\hline Vaccine-related event & $2(0)$ & $2(0)$ \\
\hline Death\| & $5(0.1)$ & $5(0.1)$ \\
\hline Discontinuation due to adverse event* & $8(0.1)$ & $4(0.1)$ \\
\hline Vaccine-related event & $5(0.1)$ & $3(0)$ \\
\hline Serious event & $3(0)$ & $1(0)$ \\
\hline Serious vaccine-related event & $1(0)$ & $0(0)$ \\
\hline
\end{tabular}

* Included in the analysis are participants who underwent randomization, received at least one dose of vaccine, and had at least one follow-up visit related to the adverse event.

$\dagger$ Adverse events were reported at any time during the study.

7 Injection-site events were adverse events that were reported within 1 to 5 days after any vaccination.

$\int$ Intensities of pain and itching were defined as mild if there was an awareness of the sign or symptom but it did not interfere with usual activities, as moderate if there was enough discomfort to cause interference with usual activity, and as severe if the pain or discomfort was incapacitating, rendering the participant unable to work or carry out usual activities.

I Systemic events were adverse events that were reported within 1 to 15 days after any vaccination.

$\|$ None of the deaths were considered by the study investigators to be vaccine-related. The five deaths in the 9vHPV vaccine group were the result of suicide ( 15 days after vaccination dose 1 ), acute lymphocytic leukemia (diagnosed 27 days after vaccination dose 3 ), traffic accident (226 days after vaccination dose 3 ), hypovolemic shock and septic shock (531 days after vaccination dose 3 ), and sudden death ( 678 days after vaccination dose 3 ). The five deaths in the GHPV vaccine group were the result of accidental death (airplane accident 7 days after vaccination dose 3 ), spinal cord injury (141 days after vaccination dose 3 ), gastric adenocarcinoma (diagnosed 403 days after vaccination dose 3 ), cervical spinal cord injury (811 days after vaccination dose 3 ), and cerebral hemorrhage (1114 days after vaccination dose 3 ).

*** Study vaccination was withdrawn. 
(Fig. 1, and Fig. S4 in the Supplementary Appendix). In the per-protocol efficacy population, the incidence of high-grade cervical epithelial neoplasia, adenocarcinoma in situ, and cervical cancer related to HPV types $31,33,45$, 52, and 58 was 0.1 per 1000 person-years in the 9vHPV group and 1.5 per 1000 person-years in the qHPV group ( 1 vs. 27 cases; efficacy, 96.3\%; 95\% CI, 79.5 to 99.8) (Table 2). The incidence of persistent infection (i.e., lasting $\geq 6$ months) related to HPV-31, 33, 45, 52, and 58 in the per-protocol efficacy population was 2.1 per 1000 person-years in the 9vHPV group and 52.4 per 1000 personyears in the qHPV group (35 vs. 810 cases; efficacy, $96.0 \%$; $95 \%$ CI, 94.4 to 97.2 ) (Table 2).

\section{IMMUNOGENICITY}

Nearly $100 \%$ of participants in the per-protocol immunogenicity population underwent seroconversion to the $9 \mathrm{vHPV}$ vaccine types within 1 month after dose 3 (Table S3 in the Supplementary Appendix). (For details on the persistence of antibody responses, see Tables S3 and S4 in the Supplementary Appendix.) According to the geometric mean titer, the noninferiority of the response to the $9 \mathrm{vHPV}$ vaccine as compared with the response to the qHPV vaccine for HPV-6, 11, 16, and 18 was established at 1 month after dose 3 (Table 3 ). Numerically, the ratios for geometric mean titer for HPV types $6,11,16$, and 18 ranged from 0.80 to 1.19 , with ratios close to 1 for HPV- 6 and 16, greater than 1 for HPV-18, and lower than 1 for HPV-11. The noninferiority of the seroconversion percentages for the 9vHPV vaccine relative to the qHPV vaccine for HPV types $6,11,16$, and 18 was established 1 month after dose 3 (Table 3). The number of cases of infection and disease associated with HPV-6, 11, 16, and 18 was similar in the 9vHPV group and the $\mathrm{qHPV}$ vaccine group (Table S5 in the Supplementary Appendix).

\section{ADVERSE EVENTS}

The recipients of the $9 \mathrm{vHPV}$ vaccine were more likely than the recipients of the qHPV vaccine to have adverse events related to the injection site $(90.7 \%$ vs. $84.9 \%)$, with the most common events (incidence $\geq 2 \%$ ) being pain, swelling, erythema, and pruritus (Table 4); more than $90 \%$ of these events were mild to moderate in intensity. Events of severe intensity were more common in the $9 \mathrm{vHPV}$ group. The frequency of systemic adverse events was generally similar in the two groups $-55.8 \%$ in the $9 \mathrm{vHPV}$ vaccine group and $54.9 \%$ in the $\mathrm{qHPV}$ vaccine group. The most common systemic adverse events related to vaccination (incidence $\geq 2 \%$ ) were headache, pyrexia, nausea, dizziness, and fatigue. Less than $0.1 \%$ of participants discontinued study vaccination because of a vaccine-related adverse event. All the serious adverse events are listed according to system organ class in Tables S6 and S7 in the Supplementary Appendix. Pregnancy was reported in 1192 participants in the 9vHPV group and 1129 participants in the qHPV group, and information on outcomes was available for approximately $85 \%$ of these pregnancies (Table S8 in the Supplementary Appendix). The proportions of participants with live births, difficulty with delivery, spontaneous abortions, and late fetal deaths were similar in the two groups. Congenital anomalies were reported in a total of 32 infants and 9 fetuses (20 in the 9vHPV group and 21 in the qHPV group). No congenital anomaly was reported in the case of pregnancies with an estimated date of conception that was within 30 days before or after any vaccination (these pregnancies represented approximately $8 \%$ of the total number of pregnancies with known outcomes).

\section{DISCUSSION}

The results of this study showed that the 9vHPV vaccine prevented cervical, vulvar, and vaginal disease and persistent infection associated with HPV-31, 33, 45, 52, and 58. Antibody responses to HPV-6, 11, 16, 18 among participants who received the $9 \mathrm{vHPV}$ vaccine were noninferior to those among participants who received the qHPV vaccine. The incidence of disease related to HPV$6,11,16$, and 18 was similar in the two vaccine groups. Therefore, we infer that the efficacy of the 9vHPV vaccine against disease related to HPV-6, 11,16 , and 18 is similar to that of the qHPV vaccine. The rate of clinical adverse events was generally similar in the two vaccine groups. The frequency of adverse events related to the injection site was higher in the 9vHPV group than in the qHPV group. This result was anticipated, since the amounts of HPV viruslike particle antigens and AAHS adjuvant are higher in the $9 \mathrm{vHPV}$ vaccine than in the $\mathrm{qHPV}$ vaccine. Most adverse events related to the injection site were 
mild or moderate in intensity. Few participants discontinued study vaccination because of a vaccine-related adverse event.

Measures were taken to enhance the accuracy, reproducibility, and generalizability of our findings. Genital inspections, biopsies of suspicious external genital lesions, cytologic screening, and colposcopy with biopsy were performed regularly and frequently to obtain a high level of sensitivity for HPV-related lesions. A panel of expert pathologists who were unaware of the group assignments was enlisted to ensure the diagnostic accuracy of conditions designated as study end points. To ensure a high level of specificity, diagnoses required detection of HPV DNA in tissue sections. Generalizability was enhanced by enrollment of a diverse population of participants from developed and developing countries and by use of standard Pap screening. Prophylactic efficacy was similar across all regions and all ethnic and racial groups. The examination procedures used and the pathologists participating in this study were the same as those in the qHPV vaccine program. ${ }^{14,15}$

The modified intention-to-treat analyses of the efficacy of the 9vHPV vaccine against diseases associated with the vaccine HPV types revealed that all cases of high-grade disease detected in the 9vHPV group occurred in participants who were HPV-infected at baseline, which underscores the importance of vaccination before exposure to HPV. Both the 9vHPV and $\mathrm{qHPV}$ vaccines are prophylactic and are not expected to prevent disease in persons who are already infected with HPV. The per-protocol efficacy population in the current analysis is the most similar to the target group for prophylactic HPV vaccination.

A limitation of the current study is the lack of a placebo control group. Given the efficacy of HPV L1 viruslike particle-based vaccination, few disease end points associated with HPV-6, 11, 16, and 18 were expected in either vaccine group, a fact that precluded a direct comparison of the $9 \mathrm{vHPV}$ and $\mathrm{qHPV}$ vaccines for these vaccine types. Moreover, no minimum protective anti-HPV antibody titer has been identified. ${ }^{43}$ Consequently, the efficacy findings associated with the licensed qHPV vaccine were extended to the investigational 9vHPV vaccine on the basis of its noninferior immunogenicity. With respect to the end points related to HPV- $31,33,45$, 52 , and 58 , the efficacy of the $9 \mathrm{vHPV}$ vaccine was determined on the basis of comparison with the GHPV vaccine group rather than with an unvaccinated population. Since the qHPV vaccine provides some crossprotection against these HPV types (especially HPV 31), ${ }^{44,45}$ the efficacy of the 9vHPV vaccine may have been underestimated.

Follow-up was limited in duration. Studies of the qHPV vaccine have not shown any evidence of waning immunity in long-term cohorts, ${ }^{46}$ which suggests that $9 \mathrm{vHPV}$ vaccine may also offer longterm protection. Longer-term follow-up of participants vaccinated with the 9vHPV vaccine is needed to provide information on the durability of protection.

In conclusion, the results of this study showed that the prophylactic administration of 9vHPV vaccine prevented infection and disease associated with the vaccine HPV types. The effect of vaccination on the burden of cancer remains to be determined.

Supported by Merck.

Dr. Joura reports receiving fees for serving on an advisory board from Merck and Sanofi Pasteur MSD, lecture fees from Merck and Sanofi Pasteur MSD and from Roche, and grant support from Merck and GlaxoSmithKline; Dr. Giuliano, fees for serving on an advisory board and grant support through her institution from Merck; Dr. Iversen, grant support from GlaxoSmithKline and Merck; Dr. Bouchard, grant and travel support from Merck and GlaxoSmithKline; Dr. Mehlsen, lecture fees and grant support from Merck; Dr. Moreira, consulting fees from Merck; Dr. Ngan, travel support from Merck, Sharp, and Dohme; Dr. Cuzick, fees for serving on advisory boards from Abbott, Gen-Probe Hologic, and Becton Dickinson, lecture fees from GlaxoSmithKline, and grant support from Roche, Abbott, GenProbe Hologic, Becton Dickinson, and Qiagen; Dr. Garland, fees for board membership and travel support from Merck, consulting fees from Merck and Sanofi Pasteur, lecture fees from Merck, Sanofi Pasteur, and GlaxoSmithKline, and grant support from Merck, GlaxoSmithKline, and CSL Behring; Dr. Huh, fees for serving on an advisory board from Merck; and Dr. Kjaer, lecture fees and fees for serving on an expert committee from Sanofi Pasteur MSD, fees for the postlicensure monitoring of the effects of HPV vaccination from Merck, and grant support from the Aragon Foundation and Merck. Drs. Bautista, Chan, and Chen, Ms. Moeller, Mr. Ritter, Dr. Vuocolo, and Dr. Luxembourg report being employees of and holding stock or stock options in Merck; and Dr. Gesser reports being a former employee of and holding stock and stock options in Merck and being a current employee of and holding stock options in Sanofi Pasteur. No other potential conflict of interest relevant to this article was reported.

Disclosure forms provided by the authors are available with the full text of this article at NEJM.org.

We thank the study investigators, study-site personnel, and the members of the V503 scientific advisory committee, the pathology panel, and the external data and safety monitoring committee, all of whom are listed in the Supplementary Appendix. 
APPENDIX

The authors' full names and academic degrees are as follows: Elmar A. Joura, M.D., Anna R. Giuliano, Ph.D., Ole-Erik Iversen, M.D., Celine Bouchard, M.D., Constance Mao, M.D., Jesper Mehlsen, M.D., Edson D. Moreira, Jr., M.D., Yuen Ngan, M.D., Lone Kjeld Petersen, M.D., Eduardo Lazcano-Ponce, M.D., Punnee Pitisuttithum, M.D., Jaime Alberto Restrepo, M.D., Gavin Stuart, M.D., Linn Woelber, M.D., Yuh Cheng Yang, M.D., Jack Cuzick, Ph.D., Suzanne M. Garland, M.D., Warner Huh, M.D., Susanne K. Kjaer, M.D., Oliver M. Bautista, Ph.D., Ivan S.F. Chan, Ph.D., Joshua Chen, Ph.D., Richard Gesser, , M.D., Erin Moeller, M.P.H., Michael Ritter, B.A., Scott Vuocolo, Ph.D., and Alain Luxembourg, M.D., Ph.D., for the Broad Spectrum HPV Vaccine Study

The authors' affiliations are as follows: the Medical University of Vienna, Comprehensive Cancer Center, Vienna (E.A.J.); Moffitt Cancer Center, Tampa, FL (A.R.G.); Department of Clinical Medicine, University of Bergen-Haukeland University Hospital, Bergen, Norway (O.-E.I.); Université Laval, Québec, QC (C.B.), and University of British Columbia, Vancouver (G.S.) — both in Canada; University of Washington, Seattle (C.M.); Coordinating Research Center, Frederiksberg Hospital, University of Copenhagen (J.M.), and Danish Cancer Society Research Center and Department of Gynecology, Rigshospitalet (S.K.K.) — all in Copenhagen; Associação Obras Sociais Irmã Dulce and Oswaldo Cruz Foundation, Brazilian Ministry of Health, Bahia, Brazil (E.D.M.); University of Hong Kong, Hong Kong (Y.N.); Aarhus University Hospital, Department of Obstetrics and Gynecology, Aarhus, Denmark (L.K.P.); Instituto Nacional de Salud Pública, Cuernavaca, Morelos, Mexico (E.L.-P.); Faculty of Tropical Medicine, Mahidol University, Nakhon Pathom, Thailand (P.P.); Investigación Clínica, Medellín, Colombia (J.A.R.); Department of Gynecology, University Medical Center Hamburg-Eppendorf, Hamburg, Germany (L.W.); Mackay Memorial Hospital, Taipei, Taiwan (Y.C.Y.); Wolfson Institute of Preventive Medicine, London (J. Cuzick); Royal Women's Hospital, University of Melbourne and Murdoch Childrens Research Institute, Parkville, VIC, Australia (S.M.G.); Division of Gynecologic Oncology, University of Alabama, Birmingham (W.H.); and Merck, Whitehouse Station, NJ (O.M.B., I.S.F.C., J. Chen, R.G., E.M., M.R., S.V., A.L.).

REFERENCES

1. Walboomers JM, Jacobs MV, Manos $\mathrm{MM}$, et al. Human papillomavirus is a necessary cause of invasive cervical cancer worldwide. J Pathol 1999;189:12-9.

2. de Sanjose S, Quint WG, Alemany L, et al. Human papillomavirus genotype attribution in invasive cervical cancer: a retrospective cross-sectional worldwide study. Lancet Oncol 2010;11:1048-56.

3. Srodon M, Stoler MH, Baber GB, Kurman RJ. The distribution of low and highrisk HPV types in vulvar and vaginal intraepithelial neoplasia (VIN and VaIN). Am J Surg Pathol 2006;30:1513-8.

4. de Sanjosé S, Alemany L, Ordi J, et al. Worldwide human papillomavirus genotype attribution in over 2000 cases of intraepithelial and invasive lesions of the vulva. Eur J Cancer 2013;49:3450-61.

5. Hatch KD. A3. Vaginal intraepithelial neoplasia (VAIN). Int J Gynaecol Obstet 2006;94:Suppl 1:S40-S43.

6. Palefsky JM. Anal squamous intraepithelial lesions: relation to HIV and human papillomavirus infection. J Acquir Immune Defic Syndr 1999;21:Suppl 1:S42S48.

7. Picconi MA, Eiján AM, Distéfano AL, et al. Human papillomavirus (HPV) DNA in penile carcinomas in Argentina: analysis of primary tumors and lymph nodes. J Med Virol 2000;61:65-9.

8. Chaturvedi AK. Beyond cervical cancer: burden of other HPV-related cancers among men and women. J Adolesc Health 2010;46:Suppl:S20-S26.

9. Kjaer SK, Tran TN, Sparen P, et al. The burden of genital warts: a study of nearly 70,000 women from the general female population in the 4 Nordic countries. J Infect Dis 2007;196:1447-54.

10. Garland SM, Steben M, Sings HL, et al. Natural history of genital warts: analysis of the placebo arm of 2 randomized phase III trials of a quadrivalent human papillomavirus (types $6,11,16$, and 18) vaccine. J Infect Dis 2009;199:805-14.

11. Christensen ND. Emerging human papillomavirus vaccines. Expert Opin Emerg Drugs 2005;10:5-19.

12. Paavonen J, Jenkins D, Bosch FX, et al. Efficacy of a prophylactic adjuvanted bivalent $\mathrm{L} 1$ virus-like-particle vaccine against infection with human papillomavirus types 16 and 18 in young women: an interim analysis of a phase III double-blind, randomised controlled trial. Lancet 2007 369:2161-70.

13. Palefsky JM, Giuliano AR, Goldstone $S$, et al. HPV vaccine against anal HPV infection and anal intraepithelial neoplasia. N Engl J Med 2011;365:1576-85.

14. Garland SM, Hernandez-Avila M Wheeler CM, et al. Quadrivalent vaccine against human papillomavirus to prevent anogenital diseases. N Engl J Med 2007; 356:1928-43.

15. FUTURE II Study Group. Quadrivalent vaccine against human papillomavirus to prevent high-grade cervical lesions. N Engl J Med 2007;356:1915-27.

16. Joura EA, Leodolter S, HernandezAvila M, et al. Efficacy of a quadrivalent prophylactic human papillomavirus (types $6,11,16$, and 18) L1 virus-like-particle vaccine against high-grade vulval and vaginal lesions: a combined analysis of three randomised clinical trials. Lancet 2007;369: 1693-702.

17. Giuliano AR, Palefsky JM, Goldstone $S$, et al. Efficacy of quadrivalent HPV vaccine against HPV Infection and disease in males. N Engl J Med 2011;364:401-11.

18. Brotherton JM, Fridman M, May CL, Chappell G, Saville AM, Gertig DM. Early effect of the HPV vaccination programme on cervical abnormalities in Victoria, Australia: an ecological study. Lancet 2011; 377:2085-92.

19. Powell SE, Hariri S, Steinau M, et al.
Impact of human papillomavirus (HPV) vaccination on HPV 16/18-related prevalence in precancerous cervical lesions. Vaccine 2012;31:109-13.

20. Baldur-Felskov B, Dehlendorff C, Munk C, Kjaer SK. Early impact of human papillomavirus vaccination on cervical neoplasia-nationwide follow-up of young Danish women. J Natl Cancer Inst 2014; 106:djt460.

21. Crowe E, Pandeya N, Brotherton JM, et al. Effectiveness of quadrivalent human papillomavirus vaccine for the prevention of cervical abnormalities: case-control study nested within a population based screening programme in Australia. BMJ 2014;348:g1458.

22. Tabrizi SN, Brotherton JM, Kaldor JM, et al. Fall in human papillomavirus prevalence following a national vaccination program. J Infect Dis 2012;206:1645-51. 23. Kahn JA, Brown DR, Ding L, et al. Vaccine-type human papillomavirus and evidence of herd protection after vaccine introduction. Pediatrics 2012;130(2):e249e256.

24. Markowitz LE, Hariri S, Lin C, et al. Reduction in human papillomavirus (HPV) prevalence among young women following HPV vaccine introduction in the United States, National Health and Nutrition Examination Surveys, 2003-2010. J Infect Dis 2013;208:385-93.

25. Read TR, Hocking JS, Chen MY, Donovan B, Bradshaw CS, Fairley CK. The near disappearance of genital warts in young women 4 years after commencing a national human papillomavirus (HPV) vaccination programme. Sex Transm Infect 2011;87:544-7.

26. Bauer HM, Wright G, Chow J. Evidence of human papillomavirus vaccine effectiveness in reducing genital warts: an analysis of California public family planning administrative claims data, 
2007-2010. Am J Public Health 2012;102 833-5.

27. Leval A, Herweijer E, Arnheim-Dahlström L, et al. Incidence of genital warts in Sweden before and after quadrivalent human papillomavirus vaccine availability. J Infect Dis 2012;206:860-6.

28. Baandrup L, Blomberg $M$, Dehlendorff C, Sand C, Andersen KK, Kjaer SK. Significant decrease in the incidence of genital warts in young Danish women after implementation of a national human papillomavirus vaccination program. Sex Transm Dis 2013;40:130-5.

29. Nsouli-Maktabi H, Ludwig SL, Yerubandi UD, Gaydos JC. Incidence of genital warts among U.S. service members before and after the introduction of the quadrivalent human papillomavirus vaccine. MSMR 2013;20:17-20.

30. Arnheim-Dahlström L, Pasternak B, Svanström H, Sparén P, Hviid A. Autoimmune, neurological, and venous thromboembolic adverse events after immunisation of adolescent girls with quadrivalent human papillomavirus vaccine in Denmark and Sweden: cohort study. BMJ 2013;347: f5906.

31. Block SL, Brown DR, Chatterjee A, et al. Clinical trial and post-licensure safety profile of a prophylactic human papillomavirus (types $6,11,16$, and 18) 11 viruslike particle vaccine. Pediatr Infect Dis 2010;29:95-101.

32. Slade BA, Leidel L, Vellozzi C, et al. Postlicensure safety surveillance for quadrivalent human papillomavirus recombinant vaccine. JAMA 2009;302:750-7.
33. Gee J, Naleway A, Shui I, et al. Monitoring the safety of quadrivalent human papillomavirus vaccine: findings from the Vaccine Safety Datalink. Vaccine 2011;29: 8279-84.

34. Chao C, Klein NP, Velicer CM, et al. Surveillance of autoimmune conditions following routine use of quadrivalent human papillomavirus vaccine. J Intern Med 2012;271:193-203.

35. Klein NP, Hansen J, Chao C, et al. Safety of quadrivalent human papillomavirus vaccine administered routinely to females. Arch Pediatr Adolesc Med 2012; 166:1140-8.

36. Grimaldi-Bensouda L, Guillemot D, Godeau B, et al. Autoimmune disorders and quadrivalent human papillomavirus vaccination of young female subjects. J Intern Med 2014;275:398-408.

37. Macartney $\mathrm{KK}$, Chiu C, Georgousakis M, Brotherton JM. Safety of human papillomavirus vaccines: a review. Drug Saf 2013;36:393-412.

38. Schiller JT, Castellsagué X, Garland SM. A review of clinical trials of human papillomavirus prophylactic vaccines. Vaccine 2012;30:Suppl 5:F123-F138.

39. Joura EA, Ault KA, Bosch FX, et al. Attribution of 12 high-risk human papillomavirus genotypes to infection and cervical disease. Cancer Epidemiol Biomarkers Prev 2014;23:1997-2008.

40. Serrano B, Alemany L, Tous S, et al. Potential impact of a nine-valent vaccine in human papillomavirus related cervical disease. Infect Agent Cancer 2012;7:38.

41. Roberts C, Green T, Hess E, et al. De- velopment of a human papillomavirus competitive luminex immunoassay for $9 \mathrm{HPV}$ types. Hum Vaccin Immunother 2014;10: 2174-103.

42. Chan ISF, Bohidar NR. Exact power and sample size for vaccine efficacy studies. Comm Stat Theory Methods 1998;27: 1305-22.

43. Joura EA, Kjaer SK, Wheeler CM, et al. HPV antibody levels and clinical efficacy following administration of a prophylactic quadrivalent HPV vaccine.don' Vaccine 2008;26:6844-51.

44. Brown DR, Kjaer SK, Sigurdsson K, et al. The impact of quadrivalent human papillomavirus (HPV; types $6,11,16$, and 18) L1 virus-like particle vaccine on infection and disease due to oncogenic nonvaccine HPV types in generally HPV-naive women aged 16-26 years. J Infect Dis 2009;199:926-35.

45. Wheeler C, Kjaer SK, Sigurdsson K, et al. The impact of quadrivalent human papillomavirus (HPV; types 6, 11, 16, and 18) L1 virus-like particle vaccine on infection and disease due to oncogenic nonvaccine HPV types in sexually active women aged 16-26 years. J Infect Dis 2009;199: 936-44.

46. Nygard $M$, Kjaer S, Dillner J, et al. Long-term effectiveness and immunogenicity of Gardasil in the Nordic countries. EUROGIN 2013, Florence, Italy, November 3-6, 2013. abstract (http://www .eurogin.com/2013/images/pdf/ EUROGIN-2013-Abstracts-Part-2.pdf).

Copyright (c) 2015 Massachusetts Medical Society. 